\title{
Hölderlin 2020 in der WLB
}

\section{Ausstellung „Aufbrüche - Abbrüche. 250 Jahre Friedrich Hölderlin”}

Am 20. März 2020 jährt sich der Geburtstag Friedrich Hölderlins zum 250. Mal. Gemessen an der begrenzten zeitgenössischen Rezeption stellt die aktuelle Bedeutung Friedrich Hölderlins, welche weltweite Dimensionen erreicht, ein erstaunliches Phänomen dar. In Deutschland wird sein Geburtstag neben dem der anderen beiden großen Jubilare Beethoven und Hegel bundesweit gefeiert. Sichtbarer Ausdruck sind die Website "Hölderlin 2020" und das 288 Seiten umfassende Programmbuch mit ca. 600 Veranstaltungen. Ein deutlicher Schwerpunkt liegt erwartungsgemäß in BadenWürttemberg.

Die Württembergische Landesbibliothek begeht das Jubiläum mit der Ausstellung „Aufbrüche - Abbrüche. 250 Jahre Friedrich Hölderlin", die im Laufe des Jahres eröffnet wird. Sie verweist mit diesem Motiv des Titels nicht nur auf eine Folge von außerordentlichen persönlichen $\mathrm{He}-$ rausforderungen, vor denen Hölderlin um 1800 stand, sondern auch auf die Erprobung neuartigen Schreibens in der Literatur. Zugleich birgt diese Periode auch politisch spannende Jahre, welche die französische und deutsche Geschichte miteinander verbindet. Dies führte zur Idee, Stuttgart und Frankreich zu zentralen Themen der Ausstellung auszuwählen.

Die Vorbereitungen dafür gehen bis ins Jahr 2017 zurück. Schon früh plante das Land Baden-Württemberg, das Hölderlinjahr 2020 im Rahmen eines großen Projekts zu fördern. So ist dem Ministerium für Wissenschaft, Forschung und Kunst sowie der BW-Stiftung ausdrücklich für die großzügige finanzielle Unterstützung zu danken. Ohne diese könnte die Ausstellung in der WLB in dieser Form nicht durchgeführt werden.

Erhebliche Verzögerungen bei der Inbetriebnahme des Erweiterungsbaus erschwerten jedoch die Vorbereitungen. Eine Ausstellung in den neuen Räumen des Erweiterungsbaus war natürlich fester Bestandteil der Planung. Es zeichnete sich jedoch schon im Herbst 2019 ab, dass eine Eröffnung im Frühjahr 2020 dort nicht möglich sein würde. Eine zeitnahe Eröffnung im Frühling (geplant war der 21. April) konnte nur im Bestandsgebäude realisiert werden. Dies erforderte aufwendige Umgestaltungen und Anpassungen an die alten Räumlichkeiten. Wiederum eine neue Situation entstand mit der Corona-Krise ab März 2020. Die Ausstellung sowie zahlreiche Begleitveranstaltungen mussten nun auf eine unbestimmte Zeit verschoben werden.

Die über 200 Exponate stammen im Wesentlichen aus dem Hölderlin-Archiv. Angesichts des großen handschriftlichen Bestandes zu Hölderlin spielte die Landesbibliothek bereits zu Beginn des 20. Jahrhunderts eine entscheidende Rolle bei der Wiederentdeckung Hölderlins. Hier stieß der Forscher Norbert von Hellingrath 1909 auf die PindarÜbertragungen Hölderlins und auf späte Hymnen, deren Bedeutung bis dahin verkannt worden waren. Er bereitete den Weg, der schließlich 1941 zur Gründung des Hölderlin-Archivs als Arbeitsstelle der Stuttgarter Ausgabe führte. Seit fast achtzig Jahren sammelt das Hölderlin-Archiv inzwischen nahezu alles zu seinem Werk und seiner international außerordentlich fruchtbaren Wirkungsgeschichte und stellt dies der Forschung und der interessierten Öffentlichkeit zur Verfügung.

Aus dessen reichen Fundus werden in der Ausstellung u.a. Handschriften, Erstdrucke, Übersetzungen, bibliophile Ausgaben, Künstlerbücher sowie audiovisuelle Medien präsentiert, um dem Publikum Hölderlin mittels von fünf ausgewählten Themenblöcken näherzubringen. Ein Begleitheft zu den fünf Stationen bildet einen guten Leitfaden durch die Ausstellung, ein reich illustrierter Katalog liefert Beiträge zu den aufgeführten Bereichen und soll sowohl die breite Öffentlichkeit als auch das Fachpublikum ansprechen.

\section{Hölderlin und Stuttgart}

Die württembergische Residenzstadt Stuttgart war für Hölderlin gerade in seinen mittleren Jahren ein wichtiger Ort. Seine Zeit in Stuttgart im Jahre 1800 steht im Rahmen eines grundlegenden Neuaufbruchs. Sein dichterisches Schaffen in Stuttgart drückt sich vor allem in seinem elegischen Werk 


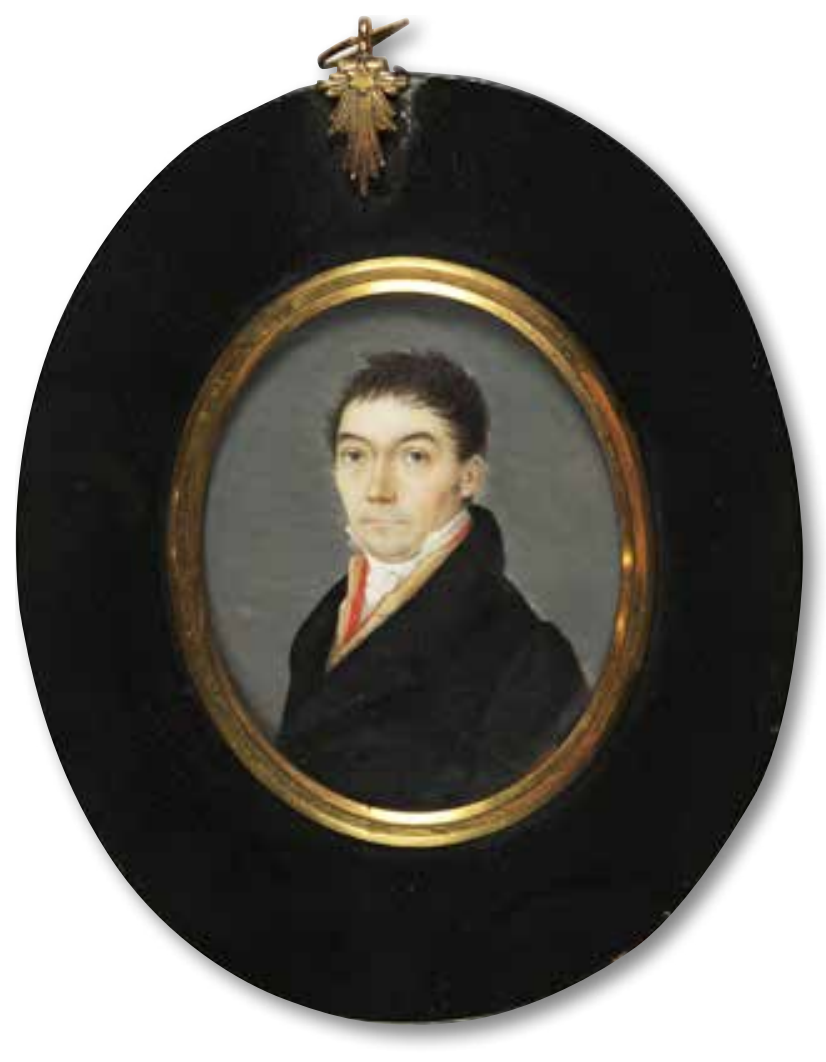

Abb. 1: Christian Landauer, Miniatur (DLA Marbach)

aus, das einen wichtigen Wendepunkt markiert hin zu einem neuen, modernen sprachlichen Ton. Über seinen Freund Christian Landauer (17691845) (Abb. 1) konnte er vielfältige Kontakte knüpfen. Landauers Haus in der Gymnasiumstraße 1,

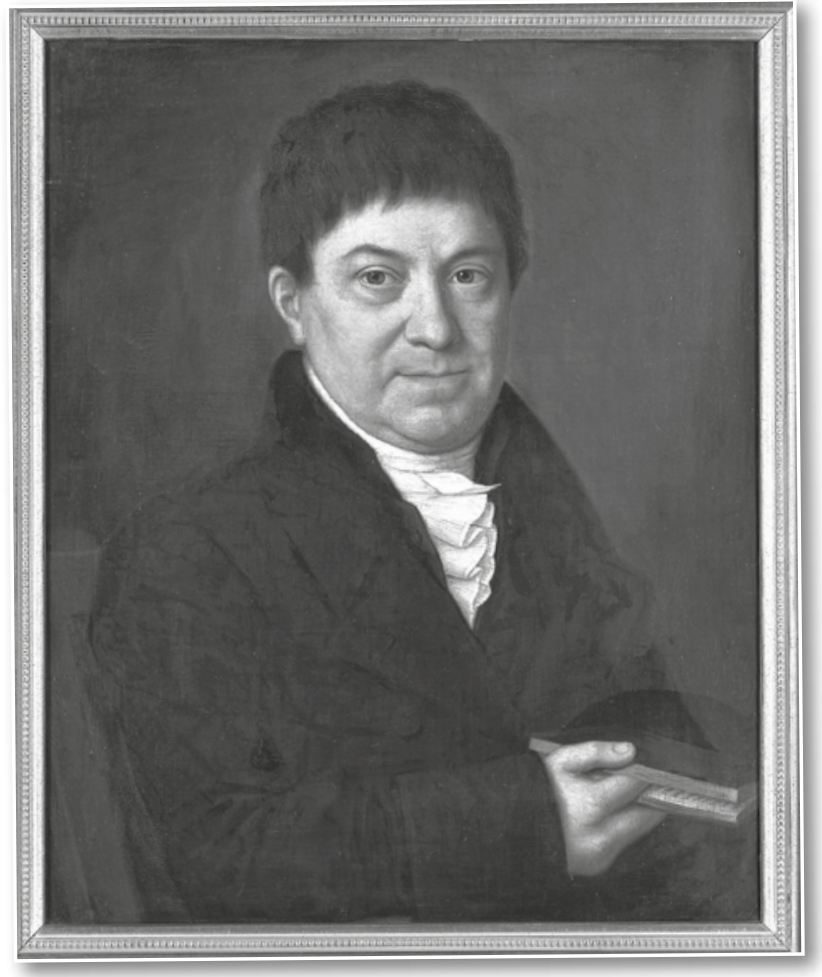

Abb. 2: Christian Ludwig Neuffer (DLA Marbach) wo er in dieser Zeit wohnte, war ein zentraler gesellschaftlicher Treffpunkt. Verschiedene Aspekte spielten hier eine Rolle. So war Hölderlin bemüht, über Kontakte zu Stuttgarter Verlegern, neue Publikationsmöglichkeiten für seine Gedichte zu finden. Die Gespräche mit Christian Ludwig Neuffer (Abb. 2), Johann Friedrich Steinkopf, Ludwig Ferdinand Huber und Friedrich Haug dokumentieren diese Bestrebungen.

Sein Stuttgarter Aufenthalt 1800 war von einer produktiven Schaffenszeit geprägt. Dies belegen vor allem die drei Gedichte "Der Gang aufs Land", "Brod und Wein" und "Stutgard", die alle einen direkten Stuttgarter Bezug aufweisen. Die erste Elegie "Der Gang aufs Land" ist Hölderlins Freund Christian Landauer gewidmet. Eine unmittelbare Beziehung zu Landauer weisen ferner die Gedichte "Das Ahnenbild" und "An Landauer" auf, beide durch das Stuttgarter Foliobuch überliefert. Während zu "Stutgard" und "Brod und Wein" neben Entwürfen auch Reinschriften im Homburger Folioheft vorliegen, existieren für die unvollendet

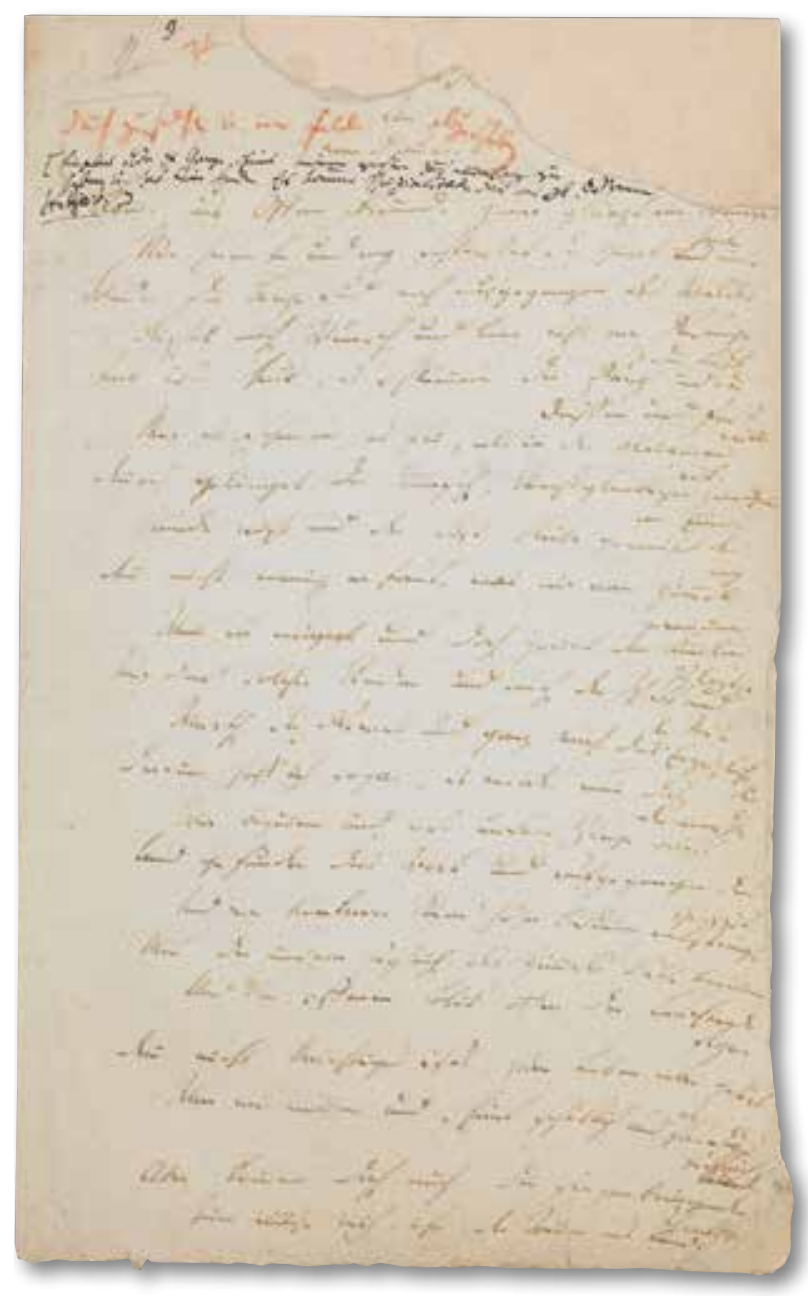

Abb. 3: Friedrich Hölderlin: "Der Gang aufs Land” (WLB, Cod.poet.et.phil.fol.63,I,9,1r) 
gebliebene Elegie „Der Gang aufs Land" nur Entwürfe wie die ausgestellte vorläufige Reinschrift, die zu Beginn Zeilen aus Justinus Kerners Hand zur Einordnung der Handschrift enthalten (Abb. 3). Aus dem Homburger Folioheft stammt neben den erwähnten Reinschriften auch Hölderlins hymnischer Entwurf „Dem Fürsten“, der das Spannungsfeld von Dichter und Fürst berührt und im brisanten politischen Kontext zu sehen ist.

Dieser politische Aspekt steht mit seiner allgemeinen Beziehung zum Fürsten im Zusammenhang. Hölderlin benötigte einen Grund, eine Rechtfertigung für seinen Stuttgarter Aufenthalt. Offiziell war er Hauslehrer und Erzieher im Hause Landauers. Doch Hölderlin sympathisierte hier mit republikanischen, zum Teil revolutionären Kreisen und geriet dadurch mehr und mehr ins Visier der

16 Obrigkeit. In diesem Umfeld standen nicht nur sein Freund Isaac von Sinclair, sondern auch der Ludwigsburger Bürgermeister Friedrich Baz. Die politischen Verwicklungen führten schließlich zum Hochverräterprozess, dem Hölderlin nur durch seine Krankheit entging.

Kurze Aufenthalte in Stuttgart rahmen auch seine Frankreichreise ein, neben Stuttgart ein Schwerpunkt der Ausstellung.

\section{Hölderlin und Frankreich}

Von Januar bis Mai 1802 hatte Hölderlin eine Stelle als Hauslehrer in Bordeaux (Abb. 4) bei dem dortigen Konsul Meyer inne. Die gesamte Frankreich-

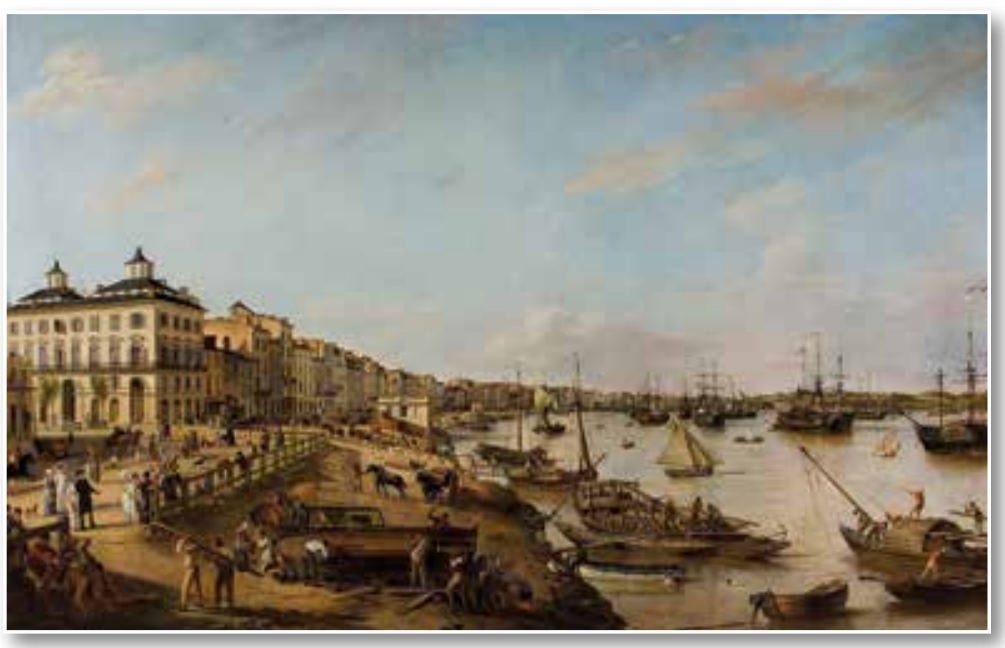

Abb. 4: "Mairie de Bordeaux" (Hafen von Bordeaux), Foto: Lysiane Gauthier

reise lässt sich als dynamischer Spannungsbogen einer extremen Auf- und Abbruchsituation verstehen. In einem Abschiedsbrief an seinen Bruder vom 4. Dezember 1801, unmittelbar vor dem Aufbruch nach Bordeaux, gesteht er bereits, wie sehr die Reise sein Innerstes berühre, dass es sich um einen großen Einschnitt in seinem Leben handele. Sie ist für inn der nächste notwendige Schritt auf der Suche nach sich selbst, nach dem eigenen Ich. Durch den Aufbruch in die Fremde soll das Eigene entdeckt werden. Als Kontrast dient hier die Welt

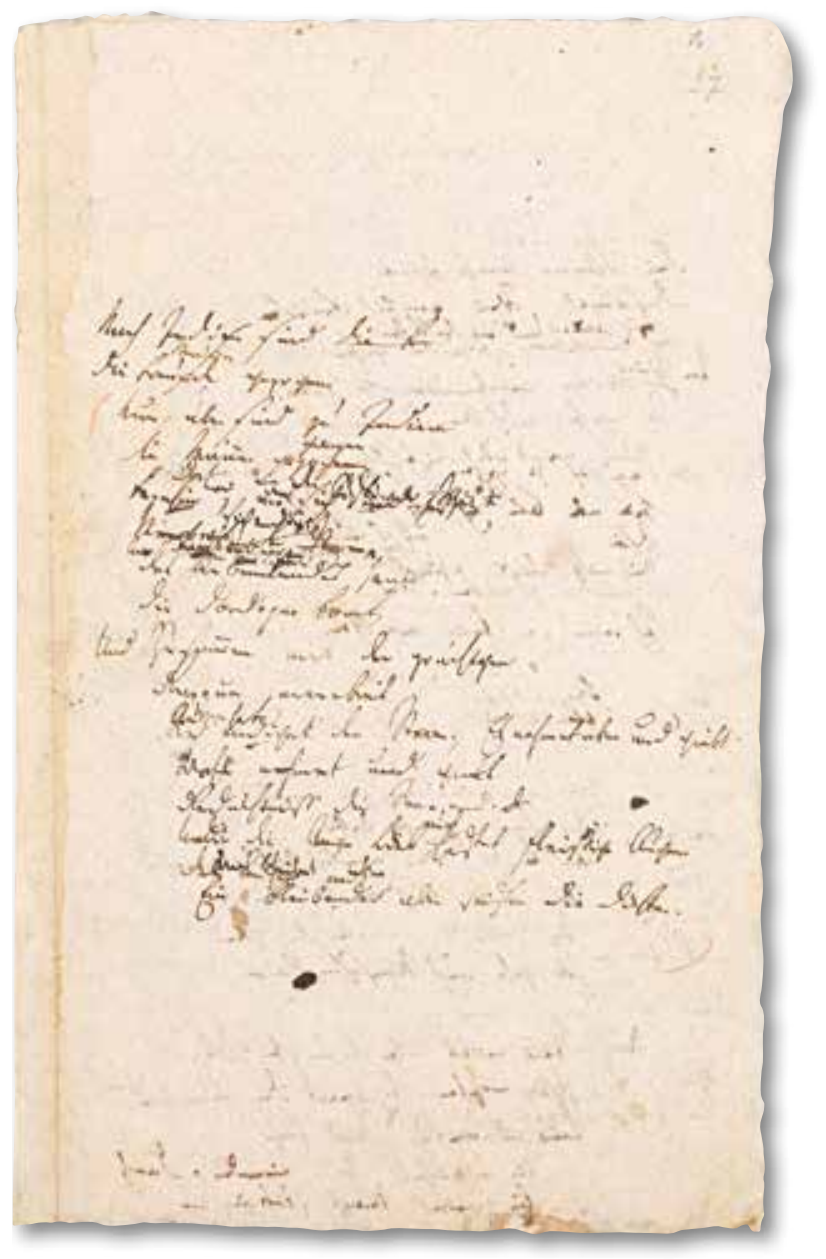

Abb. 5: Friedrich Hölderlin: "Andenken” (Homburg.H,27-28,27r)

des südwestlichen Frankreichs, wie sie später im ausgestellten Gedicht „Andenken“ (Abb. 5) im Moment des Abschieds mittels der Bordelaiser Landschaft heraufbeschworen wird. Denn auch in Bordeaux hielt es Hölderlin nicht lange aus, obwohl er zunächst sehr emphatisch der BegrüBung des Konsuls zugestimmt hatte, dass er "dort glücklich" sein werde.

Am 28. Januar angekommen, beantragte er schon Mitte Mai 1802 wieder ein Visum (Abb. 6) und brach abrupt seine Hofmeistertätigkeit ab, um sich auf den Rückweg über Paris wieder in seine Heimat zu machen, ohne dass es - wie bei früheren Stellen - zu einem Zerwürfnis oder auch nur zu Problemen mit dem Konsul und seiner Familie 


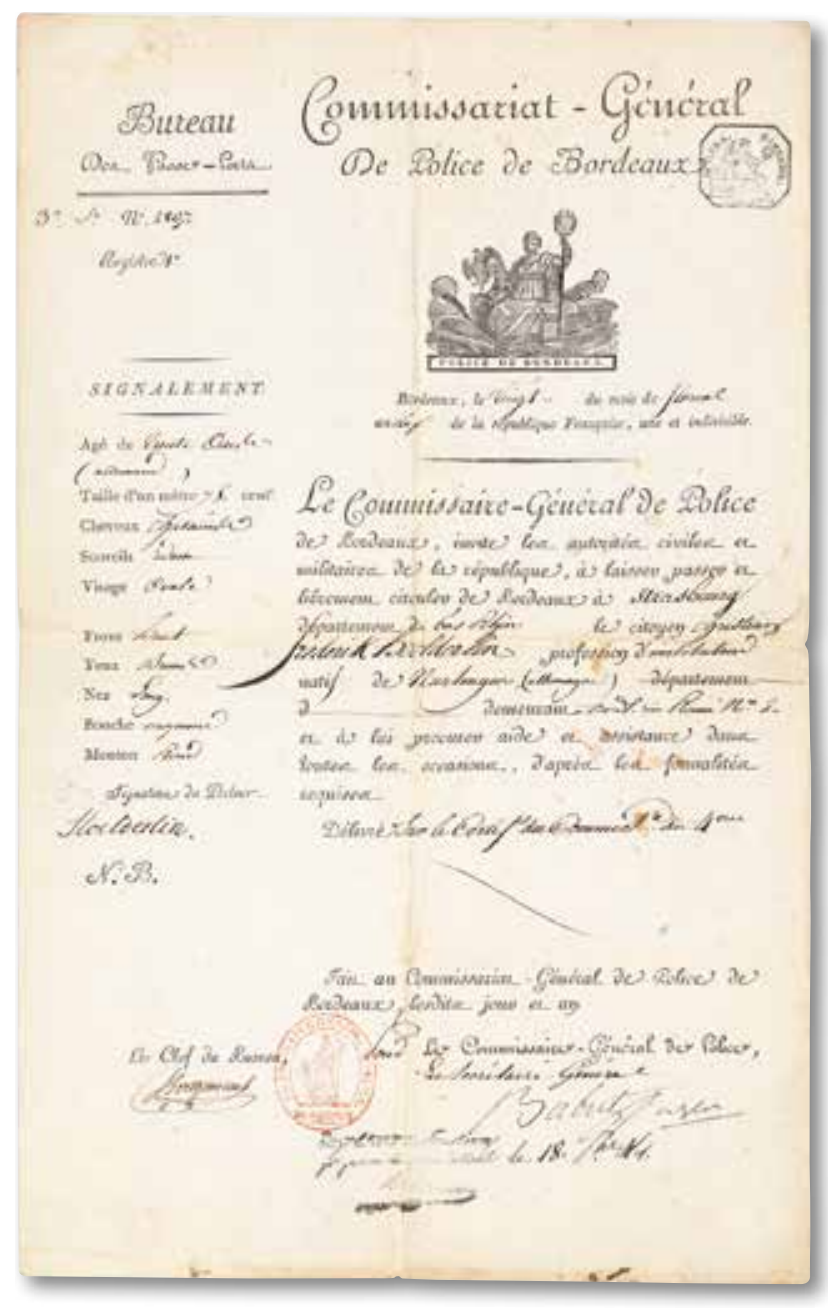

Abb. 6: Reisepass Hölderlins von Bordeaux nach Straßburg (WLB, Cod.poet.et.phil.fol.63,v,b,5)

gekommen wäre. Möglicherweise wusste er von der Krankheit Susette Gontards, die ihn zur Rückkehr angetrieben hat. Aber es zeigte sich bei Hölderlin einmal mehr eine Stimmungslage, die wie so häufig von Unruhe, Rastlosigkeit und ebenso plötzlichen wie rätselhaften Abreisen geprägt wurde. Den Hölderlin aber, der vier Wochen später in Stuttgart ankam, erlebten die Freunde als einen nicht nur geistig Zerrütteten: "Er war leichenbleich, abgemagert, von hohlem wildem Auge, langem Haar und Bart, und gekleidet wie ein Bettler," notierte Friedrich Matthison, ein Dichterfreund aus gemeinsamen Jahren im Tübinger Stift. Hölderlin befand sich körperlich und seelisch vor einem Abgrund. In jenen Tagen der Heimkehr erfuhr Hölderlin vom Tod Susette Gontards, für Hölderlin ein schwerer Schlag und Schlusspunkt seiner unglücklichen Liebe. Sie lebt fort in Hölderlins "Hyperion", den "Diotima-Gedichten" Hölderlins sowie in ihren Briefen.

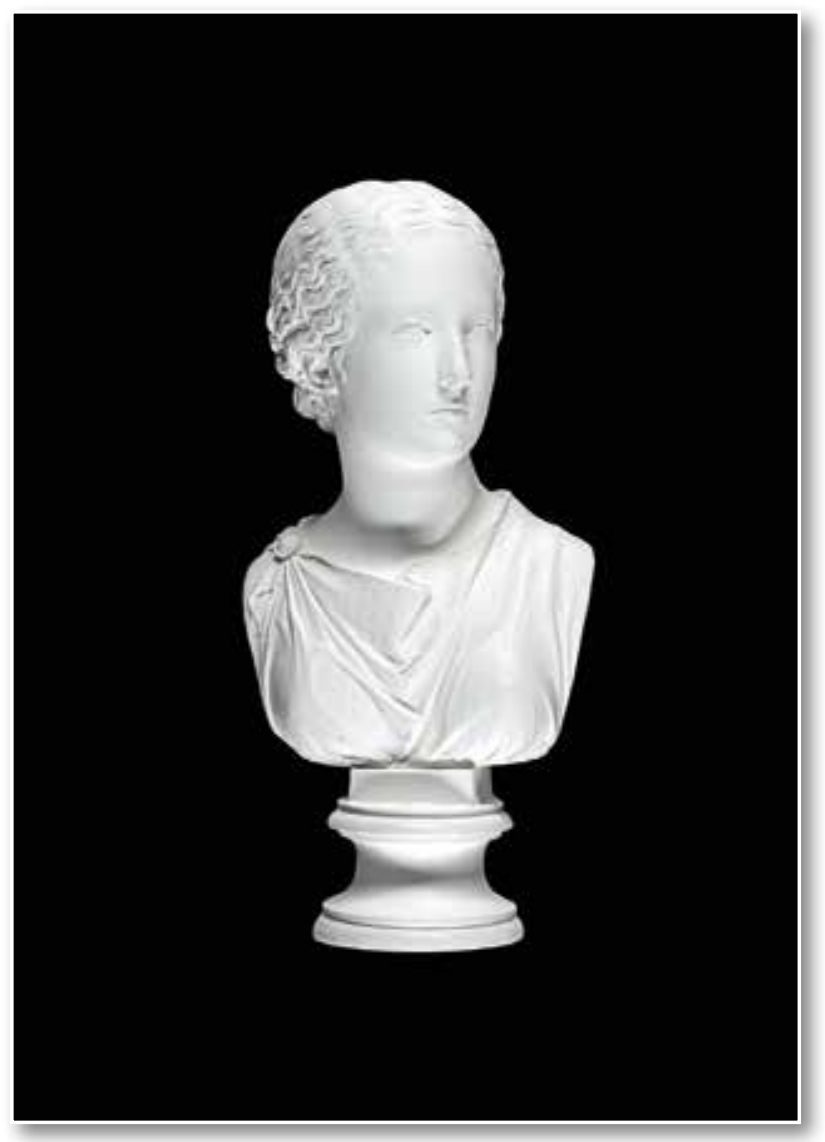

Abb. 7: Susette Gontard, Gipsbüste nach der Vorlage von Landolin Ohmacht

\section{Hölderlin und Susette Gontard}

Die Hauslehrerstelle in Frankfurt (1796-1798) stellt für Hölderlin einen positiven Wendepunkt in seinem Leben dar. In der folgenreichen Begegnung mit Susette Gontard (1769-1802) (Abb. 7) findet er schon bald ein übermächtiges Glück, das er sprachlich kaum zu fassen vermag. Sie wird zur Schlüsselgestalt von Hölderlins Leben und Werk. Real- und Idealgestalt verschmelzen schließlich miteinander: Der Dichter des „Hyperion“ setzt sie mit der Diotima der Dichtung gleich. Literarischen Ausdruck findet diese Liebe auch in zahlreichen Diotima-Gedichten. Bereits die ersten Briefe, die Hölderlin aus Frankfurt an seinen Freund Christian Ludwig Neuffer schreibt, zeugen von der ungeheuren Wirkung ihrer Begegnung. Die Bedrohung durch die französischen Truppen ermöglicht den beiden Liebenden die Reise über Kassel nach Driburg und damit einen Zeitraum des unbeschwerten Glücks. In Driburg verbringt Hölderlin zusammen mit Susette Gontard die wohl schönste Zeit seines Lebens.

Zurück in Frankfurt, schildert er in Briefen aber auch den zunehmenden Wandel seiner Gemütslage. Das für inn als ständemäßig untergeordneter 
Hofmeister empfundene demütigende Leben in der Frankfurter Gesellschaft plagt inn und überschattet das Glück. Schließlich lässt sich auch ihre Liebesbeziehung nicht länger verbergen. Es kommt zum Bruch mit dem Hausherrn. Hölderlin zieht nach Homburg zu seinem Freund Isaac von Sinclair. Die folgende Zeit ist geprägt durch monat-
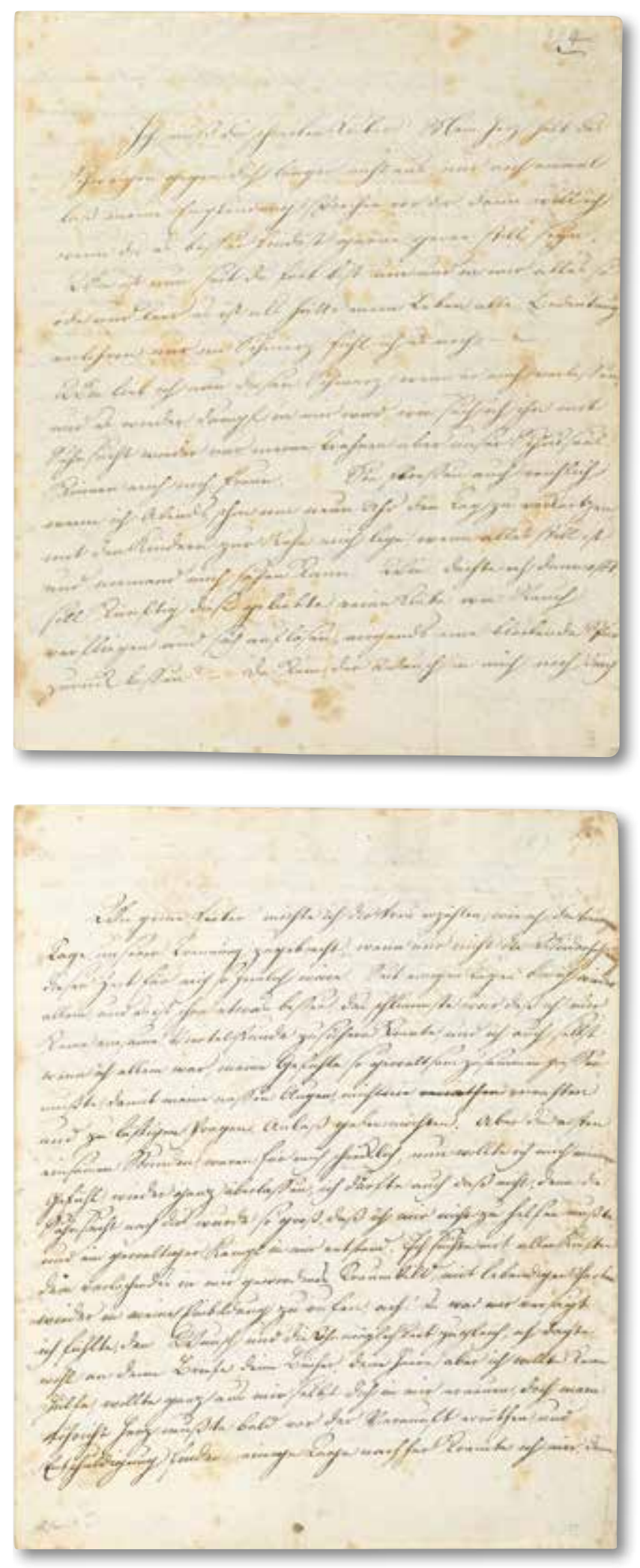

Abb. 8 und 9: Susette Gontard: Briefe an Friedrich Hölderlin (WLB, Gok-Nachlass, Cod.hist.qut.734, Mappe A, 3r und 15r) liche heimliche Treffen verbunden mit einem regen Austausch von Briefen (1798-1800). Von Hölderlin sind nur drei Briefkonzepte erhalten geblieben, von Susette Gontard sind dagegen 17 Briefe (Abb. 8 und 9) überliefert. Sie zeichnen sich durch eine besondere Schönheit aus und spiegeln die unerfüllte Liebe in großer Ausdruckskraft wider.

Diese hatte keine Zukunft. Hölderlin bricht aus dem Frankfurter Raum wieder auf, hat neue Arbeitsstellen in Stuttgart, in der Schweiz und in Bordeaux. Susette Gontard erkrankt schwer. Möglicherweise weiß er in Frankreich schon von dieser Krankheit, ein möglicher Grund seiner Heimkehr. Spätestens in Stuttgart erfährt er Anfang Juli 1802 von ihrem Tod.

Der Gok-Nachlass mit ihren Briefen wurde für diese Ausstellung digitalisiert. Dadurch werden die Briefe Susette Gontards etwa ein Jahr nach ihrem 250. Geburtstag auf neue Weise in den Fokus der Öffentlichkeit gerückt.

\section{Hölderlin digital}

Nicht nur die Digitalisierung hat in den letzten Jahren große Fortschritte erzielt, auch im Bereich der Editionswissenschaft bestehen Entwicklungen, gedruckte und handschriftliche Überlieferungsträger durch digitale Strukturen besser verknüpfbar zu machen sowie Schreibprozesse zu visualisieren. Diese Möglichkeiten werden auch mehr und mehr für Hölderlin nutzbar gemacht. Als zentrale Basis wurden zwischen 2010 und 2012 die Stuttgarter Ausgabe und der Großteil der Hölderlin-Handschriften digitalisiert. Es folgte aktuell als weiterer Teil der Digitalen Sammlungen der WLB die Digitalisierung des Gok-Nachlasses mit den Liebesbriefen von Susette Gontard. Eine wichtige Ergänzung bildet ein Projekt, das die zeitliche Dimension des Homburger Foliohefts erfasst und auf die genetische Darstellung der wichtigsten Sammelhandschrift Hölderlins zielt. Das von Dr. Gerhard Steimer durchgeführte Projekt, finanziert von der A und A Kulturstiftung, ist in das Online-Angebot der WLB aufgenommen worden. Der Leser kann hier anschaulich die Arbeitsphasen Hölderlins Schritt für Schritt mitverfolgen. Die farbliche Kennzeichnung der Phasen hilft, die einzelnen Schritte nachzuvollziehen (Abb. 10-12). Ebenso wie die digitalisierten Briefe Susette Gontards wird dieses Projekt in der Ausstellung erstmals öffentlich präsentiert. Mit diesem Ausflug in seine "Dichter- 

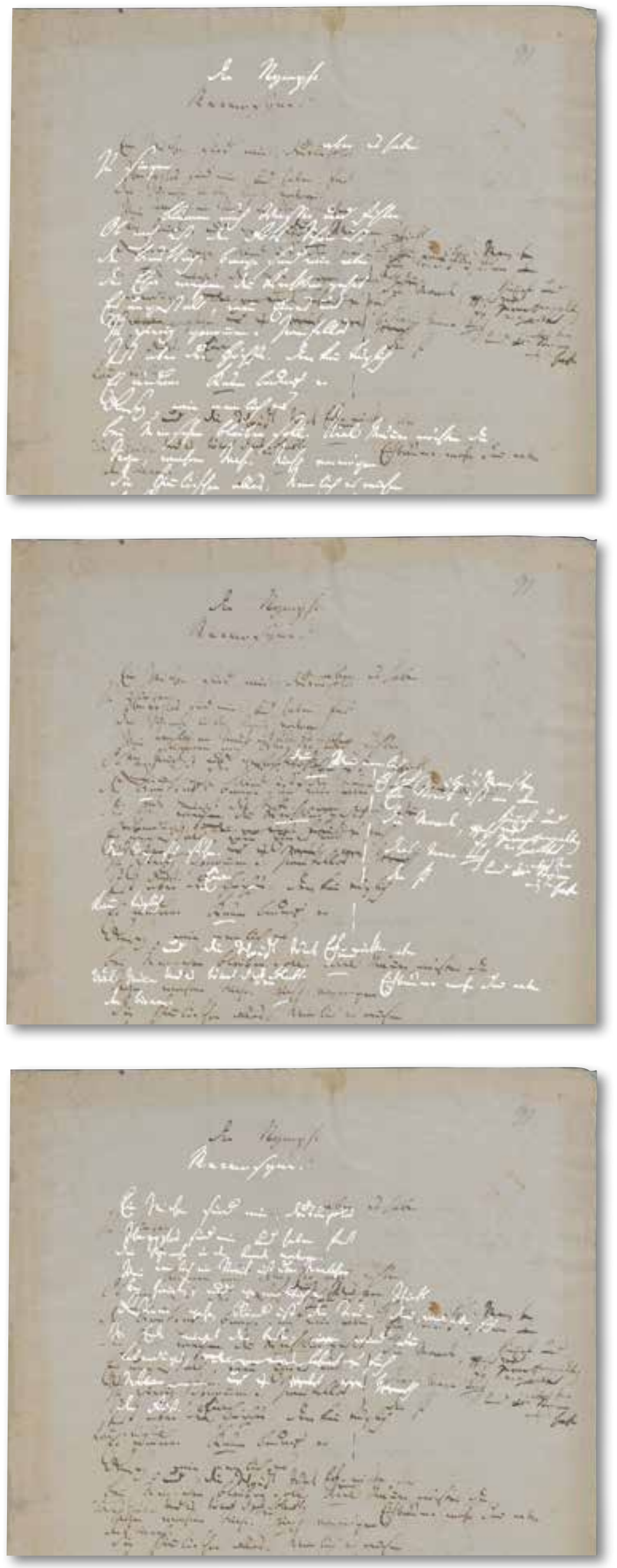

Abb. 10-12: Friedrich Hölderlin: "Mnemosyne". Bearbeitungsstufen zum Homburger Folioheft (Homburg.F,91) werkstatt" besteht die Möglichkeit, Hölderlin auf unmittelbarste Art und Weise zu lesen. Zugleich zeigt dieser Arbeitsprozess einmal mehr die Vielfalt der Lesarten, die Hölderlins Werke auszeichnen. Eine modular aufgebaute Station ermöglicht dem Publikum, sowohl die Transkriptionen ausgewählter Handschriften aufzurufen als auch die verschiedenen Facetten von „Hölderlin digital“ zu erkunden. Denn ein wichtiges Anliegen der Ausstellung ist, Hölderlin ein Stück weit lesbar zu machen, zur eigenen Auseinandersetzung mit Hölderlintexten zu ermutigen und seine eigenen Entdeckungen zu machen.

\section{Hölderlin entdecken}

Kaum ein Schriftsteller hat eine so vielfältige, aber auch so widersprüchliche Rezeption erfahren. Wir erfahren, wie zeitabhängig die frühere Rezeption und Hölderlinverehrung erscheinen, sei es in der künstlerischen Rezeption, in den Arbeiten der Wissenschaften oder in den Feierlichkeiten zu seinen Jahrestagen.

Ergänzt von zwei Audio-Stationen, in denen beispielhaft die musikalische Rezeption wie auch die Rezitation (Beispiel: Bruno Ganz) vermittelt wird, macht die Ausstellung die Rezeptionsgeschichte Hölderlins anhand von fünf Bereichen sichtbar:

1) Norbert von Hellingrath

2) Kunstrezeption (Bibliophile Ausgaben, Künstlerbücher)

3) Übersetzungen

4) Französische Rezeption

5) Gedenkfeiern 1870-1993

\section{Norbert von Hellingrath (1888-1916)}

Die außergewöhnliche Rezeption Hölderlins im 20. Jahrhundert ist ohne die aufsehenerregende Neuentdeckung durch Norbert von Hellingrath (Abb. 13) in der damaligen Königlichen Landesbibliothek in Stuttgart im Oktober 1909 kaum denkbar. Hellingraths Dissertation über Hölderlins PindarÜbertragungen sowie seine Forschungsarbeit leiteten einen Paradigmenwechsel und Neuzugang zum Spätwerk Hölderlins ein: Dieses war nicht mehr als ein Zeugnis des Wahnsinns, sondern als ein Höhepunkt seines dichterischen Schaffens zu betrachten. Die späten Hymnen Hölderlins, im 19. Jahrhundert kaum beachtet, wurden nun in einem völlig neuen Licht gesehen. Wichtig für Hellingraths Forschungen waren seine Kontakte 


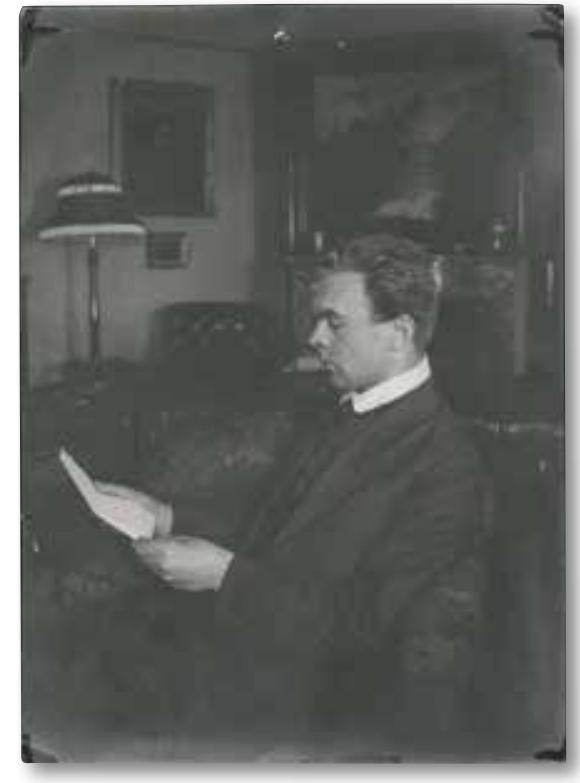

Abb.13: Norbert Hellingrath an seinem Schreibtisch Dichtung

und pflegte einen regen Briefwechsel mit ihm. Der Kontakt zu Stefan George ermöglichte Hellingrath, den neuen Fund der Pindar-Übertragungen neben seiner Dissertation auch in den von Hölderlins Spätwerk, in der Anthologie „Das Jahrhundert Goethes" im dritten Band der "Deutschen Dichtung" erscheinen konnte. Hellingraths Forschungen beschränkten sich nicht nur auf die Pindarübersetzungen und die späten Hymnen, sondern bezogen das gesamte Werk Hölderlins mit ein als Ausgangspunkt einer neuen HölderlinGesamtausgabe. Dessen erster Band erschien 1913. Die Arbeiten Hellingraths fanden jedoch mit dem Ersten Weltkrieg ein jähes Ende. Vor Verdun starb er im Jahre 1916, kurz vor Erscheinen des vierten Bandes seiner Ausgabe. Fortgeführt wurde die Edition von Friedrich Seebaß und Ludwig von Pigenot.

Vom Nachlass Hellingraths gelangte zunächst 1964 nur Teil 1 ins Hölderlin-Archiv und erst im Jahr 1983 nach dem Tod seiner Verlobten, Imma von Bodmershof, geb. Ehrenfels (1895-1982), auch der Teil 2. Das Herzstück dieses zweiten Nachlassteiles, die Briefe Hellingraths an Imma, ermöglichte erst eine präzise und differenzierte Betrachtung seines Hölderlinverständnisses.
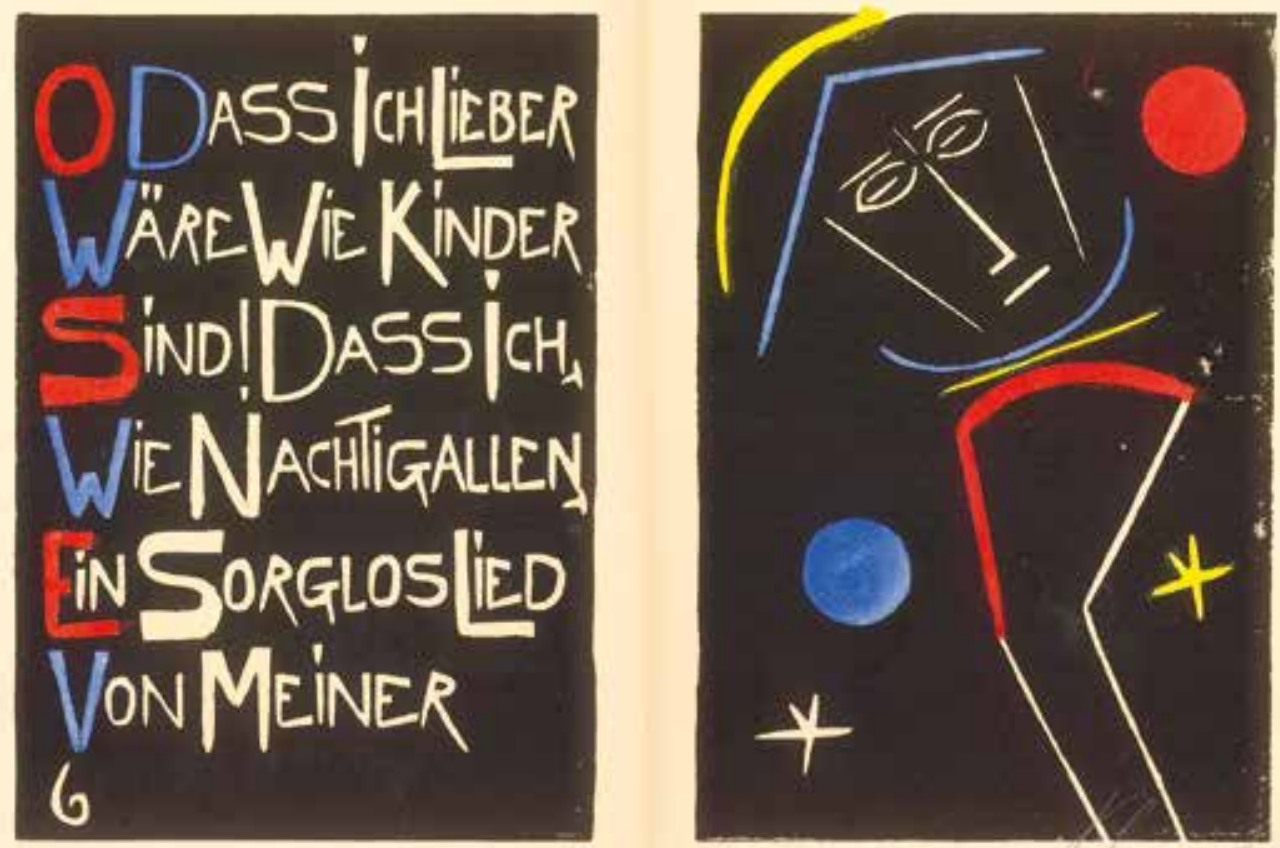

Abb. 14: Friedrich Hölderlin: Gedichte. Holzschnitte von Karl Lorenz. Turmpresse, 1927

„Blättern für die Kunst“ im Jahre 1910 zu veröffentlichen. Stefan George und Karl Wolfskehl sorgten zudem dafür, dass auch die Hymne „Wie wenn am Feiertage", ein Schlüsseltext zum Verständnis

\section{Kunstrezeption}

Das Hölderlin-Archiv besitzt über 300 künstlerische Bücher und Drucke, die sich auf Leben und Werk des Dichters beziehen. Dazu gehören neben 


\section{Narcyssen Ranunklen und Siringen aus Persien Nelken, gezogen Blumen perleniarb Und schwarz und Hyacinthen,}

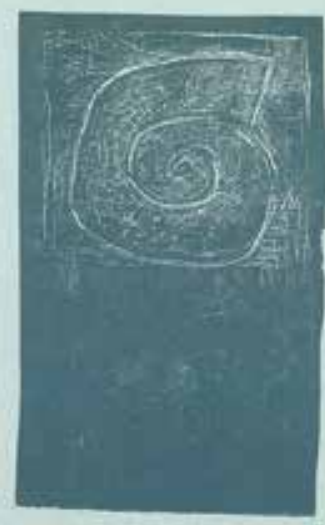

Abb. 15: Friedrich Hölderlin: Narcyssen Ranunklen... III. und Druck: Hermann Rapp. Offizin Goldene Kanne, 2013

bibliophilen Ausgaben vor allem moderne Künstlerbücher. Die bibliophilen Ausgaben in den 1920er Jahren standen unter dem Einfluss der Buchkunstbewegung Anfang des 20. Jahrhunderts. Verbunden wurden mit Blick auf die Wiedergewinnung des "schönen Buchs" Handwerk und Künstlertum, Typographie, Illustration und Einbandgestaltung. Zu den Trägern der Buchkunstbewegung gehörten insbesondere wichtige Zeitschriften (Die Jugend, Simplicissimus, Die Insel) sowie Handund Privatpressen (Abb. 14). Im Laufe des 20. Jahrhunderts trat zunehmend das Künstlerbuch als eigenständiges Kunstwerk an die Seite der bibliophilen Ausgaben. Nicht mehr Eigenschaften des "schönen Buchs", sondern das individuelle künstlerische Konzept trat in den Vordergrund. Seit Mitte der 1960er Jahre lässt sich eine erstaunliche Kontinuität und Breite der künstlerischen Rezeption beobachten.

Gerade das Offene und Fragmentarische an Hölderlins Werk schuf stetig neue Möglichkeiten einer schöpferischen künstlerischen Aneignung. Hier konnten gleichsam künstlerische Auf- und Abbrüche thematisiert werden. Zahlreiche Techniken fanden hier Anwendung. Die Bandbreite reicht von Techniken der Malerei über Druckgraphiken und Zeichnungen bis hin zu Scherenschnitten, Papiertechniken und Skulpturen. Die hier ausgestellten Beispiele können nur eine kleine Auswahl dieser Vielfalt wiedergeben. Neben Buchkünstlern wie Hermann Rapp (Abb. 15) und Robert Schwarz, die beide über einen großen Zeitraum ein bemerkens- wertes Repertoire an „Hölderlin-Büchern" hervorbrachten, treten international hochrangige Künstler wie Markus Lüpertz, der sich Hölderlin nicht nur in der Malerei nähert, sondern ebenso auf dem Gebiet der Grafik und der Bildhauerei. Jeder der vielen künstlerischen Persönlichkeiten entdeckt Hölderlin auf seine ganz eigene Weise, versucht den Charakter von Hölderlins Dichtung neu zu vergegenwärtigen.

\section{$\underline{\text { Übersetzungen }}$}

Ergänzt wird die Rezeption von der Präsentation ausgewählter Übersetzungen. Denn die Übersetzungen trugen wesentlich zu seiner außergewöhnlichen Rezeptionsgeschichte bei. Heute gilt Hölderlin als einer der am meisten übersetzten deutschen Lyriker, da er die moderne Dichtersprache maßgeblich geprägt hat.

Zu den bedeutendsten Übersetzern gehören André du Bouchet, Luis Cernuda, Michael Hamburger, Philippe Jaccottet, Carles Riba und Gustave Roud. Der Schwerpunkt der Ausstellung liegt auf den französischen Übersetzungen, da Hölderlins Rezeption in Frankreich eine Ausnahmestellung im Spektrum der internationalen Rezeption zukommt. Die Übersetzungen sind eng mit der französischen Rezeption verflochten. Französische Schriftsteller und Philosophen (Philippe Jaccottet, Jean-Pierre Lefebvre, Michel Deguy) waren gleichzeitig auch wichtige Übersetzer. Zudem gehen größere Übersetzungsprojekte häufig mit Neueditionen einher. Für eine präzise Übersetzung ist meistens 


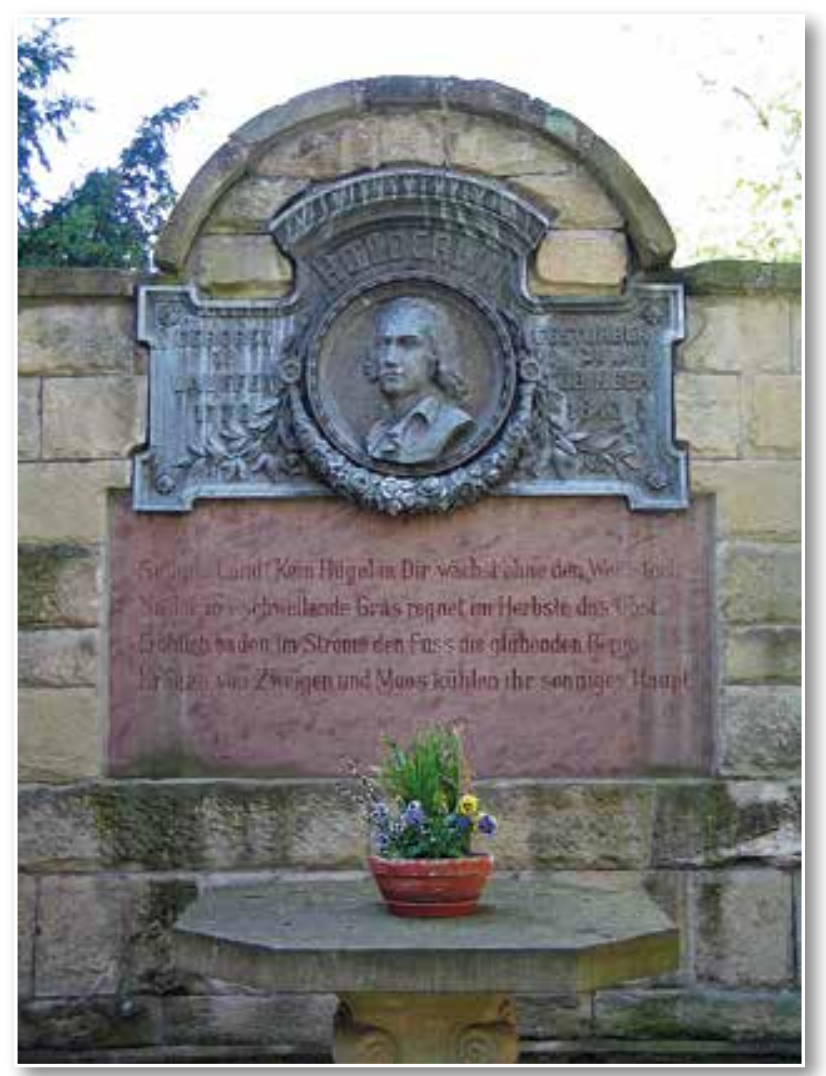

Abb. 16: Denkmal in Lauffen, 1873

die kritische Auseinandersetzung mit mehreren Hölderlin-Ausgaben nötig. Das Ergebnis ist nicht nur eine neue Übersetzung, sondern zugleich eine eigenständige, fremdsprachige Hölderlin-Edition mit umfangreichen Kommentaren (Luigi Reitani, Michael Hamburger, David Constantine, Zhengxiang $\mathrm{Gu}$ ). Eine Besonderheit stellen die polyglotten Ausgaben dar: Das Hölderlin-Archiv besitzt Ausgaben mit 30 Übersetzungen zum Gedicht „Andenken" und 69 Übersetzungen zum bekanntesten Gedicht Hölderlins, „Hälfte des Lebens”.

\section{Französische Rezeption}

Im Rahmen der internationalen Hölderlin-Rezeption nimmt die französische Rezeption eine Ausnahmestellung ein. Sie hängt unmittelbar mit der Diskussion literarischer und philosophischer Grundfragen zusammen und basiert zu einem wesentlichen Teil auf der Wirkung Martin Heideggers. Aber auch Maurice Blanchot, Jean Laplanche, Jacques Derrida, Philippe Jaccottet, Pierre Bertaux und Jean-Pierre Lefebvre haben die komplexe Rezeptionsgeschichte maßgeblich geprägt.

Neben Beispielen aus der Philosophie und Literaturtheorie beleuchtet die Ausstellung auch die Rezeption in Theater und Film. Zu nennen sind hier die Gemeinschaftsproduktionen von Jean-Marie Straub und Danièle Huillet. Sowohl der "Empedokles" als auch die Sophokles-Übersetzungen Hölderlins sind für ihre Inszenierungen richtungsweisend. Wichtig ist in diesem Kontext auch ihre Auseinandersetzung mit Bertolt Brecht, Heiner Müller und Klaus Michael Grüber.

\section{Gedenkfeiern 1870-1993}

Der tiefgreifende Wandel seiner Rezeptionsgeschichte drückt sich besonders gut in den Gedenkfeiern aus, die eine beachtliche Bandbreite aufweisen. Hier spannt sich der Bogen von regionalen Veranstaltungen bis zu großen nationalen Feiern, von sprachlich-ästhetischen Themen bis hin zu politisch-ideologischen Vereinnahmungen. Zu Hölderlins 100. Geburtstag gab es überwiegend regionale Gedenkveranstaltungen, in denen die Planung von Denkmälern im Fokus stand (Abb. 16). 50 Jahre später schafften Faktoren wie die Buchkunstbewegung und die Theaterrezeption neue Zugänge, die auch im Zusammenhang mit der Neuentdeckung Hölderlins zu sehen sind. Die zunehmende Politisierung der Hölderlin-Rezeption zeigte sich insbesondere in den Gedenkfeiern von 1943 und 1970. Stand der 100. Todestag ganz im Zeichen der nationalsozialistischen Propaganda, war beim 200. Geburtstag der Einfluss der Linken nach dem revolutionären Aufbruch von 1968 deutlich spürbar. Nötig war 1993 eine kritische Reflexion und differenzierte Analyse der Rezeptionsgeschichte, um Hölderlin von politischen Inanspruchnahmen zu lösen und den Blick zu öffnen für einen angemessenen Zugang des vielseitigen Dichters, um seine ganze Komplexität zu fassen.

\section{Begleitprogramm}

Ergänzt wird die Ausstellung durch ein attraktives kulturelles Begleitprogramm mit zahlreichen Veranstaltungen. Ein Schwerpunkt liegt dabei auf der Musik, vielleicht dem für Hölderlin bedeutendsten Rezeptionszweig. Das Hölderlin-Archiv besitzt allein weit über 1.400 Partituren. Komponisten mit bedeutenden Hölderlin-Vertonungen wie Luigi Nono, Paul Hindemith, Hanns Eisler und Hermann Reutter sind im Begleitprogramm vertreten. Viele weitere Bereiche runden das Programm ab. Folgende Veranstaltungen sind geplant: 
- HOELDER! Hymnen an die Unsterblichkeit: Konzertante Erlebnislesung mit Holly Loose (Gesang und Vorleser), Benjamin Gerlach (E-Cello), Karl Helbig (Saxofon), Silvio Schneider (Gitarre)

- HÖLDERLIN. Bilder-Klänge-VerDichtung: Intermediale Performance mit Werner Englert (Musik), Katharina Hoehler (Malerei) und Dieter E. Neuhaus (Schauspiel/Dramaturgie)

- Hans Gerhard Steimer: Friedrich Hölderlins Homburger Folioheft in diachroner Darstellung

- Vertonungen von Friedrich Hölderlin Ein Liederabend mit Peter Schöne (Bariton) und Alexander Fleischer (Klavier) in Kooperation mit der Hugo-Wolf-Akademie

- FRIEDRICH HÖLDERLIN / DICHTER SEIN. UNBEDINGT!

Buch und Regie: Hedwig Schmutte und Rolf Lambert in Ko-Produktion mit SWR und Arte

- "Hölderlin sichtbar machen“. Ein Projekt des Hölderlin-Gymnasiums Stuttgart

- Der Not ist jede Lust entsprossen - ein Konzertabend auf Spuren Hölderlins mit Susanne Stock (Akkordeon), Anne Schneider (Gesang), Urs Stämpfli (Schauspiel), Astrid Alexander (Audio)

- Rezitationsabend der Württembergischen Landesbibliothek in Kooperation mit der Akademie für gesprochenes Wort

- Hölderlin-Vertonungen von Luigi Nono und Hans Zender

Ein Konzert mit dem Kairos Quartett in Kooperation mit der Hugo-Wolf-Akademie

- "Hölderlin ausstellen“ - ein Kuratorengespräch

- Karl-Heinz Ott: Hölderlins Geister Mit dem Autor Karl-Heinz Ott und Prof. Dr. Manfred Koch

- "O Asia, das Echo von dir « - Hölderlin und Beethoven in Wort, Szene und Musik Mit Corinna Harfouch (Rezitation) und Hideyo Harada (Klavier)

\section{Ausblick}

Ein Teil der Ausstellung kann nochmals im Anschluss in der Bibliothèque nationale et universitaire in Straßburg präsentiert werden. Angesichts der Begeisterung für Hölderlin in Frankreich ist es eine schöne Frucht der vielfältigen Zusammenarbeit mit der Partnerbibliothek der WLB. Hölderlin ist ein Dichter, der uns eint.

Passend zur Ausstellung in der BNU ist vom 21. September bis 27. September eine Studienreise nach Bordeaux auf den Spuren Hölderlins geplant. Veranstaltet wird die Exkursion vom Schwäbischen Heimatbund, die Leitung der Studienfahrt hat Prof. Dr. Thomas Knubben.

Jörg Ennen 